\title{
Thermal neutrinos from hot GRB fireballs
}

\author{
Hylke B. J. Koers* \\ NIKHEF \\ PO Box 41882, 1009 DB Amsterdam, The Netherlands \\ and \\ University of Amsterdam, Amsterdam, The Netherlands \\ E-mail: hkoers@nikhef.nl
}

Ralph A.M.J. Wijers

Astronomical Institute 'Anton Pannekoek'

Faculty of Science, University of Amsterdam

Kruislaan 403, 1098 SJ Amsterdam, The Netherlands

\begin{abstract}
We consider the physics of neutrinos in a fireball, i.e. a tightly coupled plasma of photons, positrons and electrons. Such a fireball is believed to form in the first stages of a gamma-ray burst. We assume the fireball is radiation-dominated and spherically symmetric. Energy considerations limit the allowed baryon density, from which it follows that the neutrino physics is dominated by leptonic processes. We find that, for quite general initial conditions, neutrinos start out in thermodynamic equilibrium with the fireball and follow the usual hydrodynamical evolution. As the fireball cools, the plasma becomes transparent to neutrinos which subsequently decouple from the plasma. Although a sizable fraction of the total energy is carried away, the detection possibility of these neutrino bursts is limited due to the isotropic outflow and the relatively low mean energy of approximately $60 \mathrm{MeV}$.
\end{abstract}

International Europhysics Conference on High Energy Physics

July 21st - 27th 2005

Lisboa, Portugal

${ }^{*}$ Speaker. 


\section{Introduction}

The current paradigm on long gamma-ray bursts (GRBs) is that they are initiated by the violent collapse of a massive star (see e.g. [8]). In this process, a black hole - accretion disk system is formed, which stores huge amounts of energy. Assuming that a fair fraction of the gravitational energy is liberated into a volume contained within a few Schwarzschild radii, one expects the formation of a fireball, a photon-electron-positron plasma with a small baryonic load [1]. Radiation pressure accelerates the fireball to high Lorentz factors. This process converts thermal energy to kinetic energy of the baryons, which is again dissipated by shock acceleration in the optically thin region far away from the central object. This results in the observed gamma-ray bursts.

The physics of these photon-electron-positron fireballs was explored by Cavallo and Rees [1]. With the cosmological distances and higher energies presently believed to be involved, the fireball could be hot enough to contain neutrinos ${ }^{1}$. We investigate the role of neutrinos in the dynamical evolution of the fireball and the expected neutrino emission.

\section{The fireball}

The parameter space of a generic fireball can be divided into three regions: region I where the fireball contains neutrinos of all flavors; region II where it contains only electron-neutrinos; and region III where all neutrinos are decoupled. In thermodynamic equilibrium, the energy density and temperature are related through $\mathscr{E} / V=g a T^{4}$, where $a$ is the radiation constant and $g_{\mathrm{I}}=43 / 8$; $g_{\mathrm{II}}=29 / 8 ; g_{\mathrm{III}}=11 / 4$. We assume a spherical configuration and adopt the following reference values for the initial fireball energy, radius and temperature:

$$
\mathscr{E}_{*}=10^{52} \mathrm{erg}, \quad R_{*}=10^{6.5} \mathrm{~cm}, \quad T_{*}=2.1 \times 10^{11} \mathrm{~K}=17.9 / k_{B} \mathrm{MeV} .
$$

Typical number densities for particles in thermal equilibrium are of the order $n \sim 10^{35} \mathrm{~cm}^{-3}$. The nucleon ${ }^{2}$ density is restricted by energy considerations: we require $1 \mathrm{TeV}$ per nucleon for acceleration to sufficiently large Lorentz factors. We take this upper limit on the density as the reference value: $n_{B, *}=5 \times 10^{31} \mathrm{~cm}^{-3}$. Because of overall charge neutrality, the net electron density $\Delta n_{e}:=n_{e^{-}}-n_{e^{+}}$equals the proton density. The low nucleon density then implies that $\Delta n_{e} \ll n_{e^{-}}+n_{e^{+}}$, so that the electron chemical potential is very small: $\mu_{e} /\left(k_{B} T\right) \ll 1$.

\section{Fireball neutrino physics}

The neutrino physics in this environment is dominated by leptonic processes (for a more elaborate discussion on the various processes, see [5]). The dominant neutrino production process is electron-positron pair annihilation $e^{-}+e^{+} \rightarrow v+\bar{v}$, with total emissivity [2, 4]

$$
Q_{\text {pair }}=3.6 \times 10^{33}\left(T_{11}\right)^{9} \mathrm{erg} \mathrm{s}^{-1} \mathrm{~cm}^{-3}, \quad \text { where } T_{11}=T / 10^{11} \mathrm{~K} .
$$

The neutrino mean free path (mfp) is set by scattering off electrons and positrons [7]:

$$
\lambda^{(\mathrm{e})}=3.7 \times 10^{6}\left(T_{11}\right)^{-5} \mathrm{~cm}, \quad \lambda^{(\mu, \tau)}=1.6 \times 10^{7}\left(T_{11}\right)^{-5} \mathrm{~cm} .
$$

\footnotetext{
${ }^{1}$ We will generally use 'neutrinos' for 'neutrinos and antineutrinos'.

${ }^{2}$ Because the temperature is higher than typical binding energies, all nuclei are dissociated into nucleons.
} 


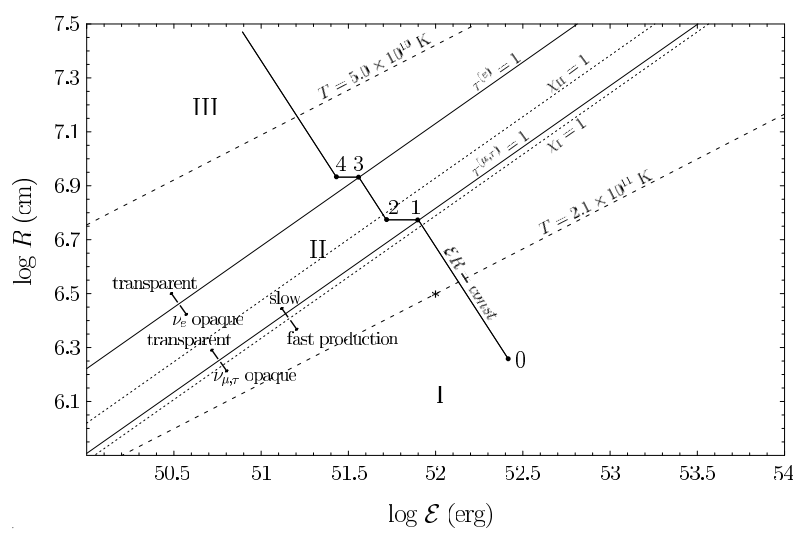

Figure 1: The parameter space of the fireball. The solid lines show the $\chi_{\mathrm{I}}=1, \chi_{\mathrm{II}}=1, \tau^{(\mu, \tau)}=1$ and $\tau^{(\mathrm{e})}=1$ contours; the dotted lines are isotemperature curves. The $*$ denotes the reference point of eq. (2.1).

The mfps for neutrinos and for antineutrinos are virtually equal because of the equal amounts of electrons and positrons. We express the neutrino creation rate in terms of the parameter $\chi=t_{c} / t_{e}$, where $t_{c}=\mathscr{E} /(V Q)$ is the cooling timescale and $t_{e}=R / c_{s}$ is the expansion timescale:

$$
\chi=3.7 \times 10^{-3} g^{9 / 4}\left(\mathscr{E}_{52}\right)^{-5 / 4}\left(R_{6.5}\right)^{11 / 4}, \quad \mathscr{E}_{52}=\mathscr{E} / 10^{52} \mathrm{erg}, \quad R_{6.5}=R / 10^{6.5} \mathrm{~cm} .
$$

We used $c_{s}=c / \sqrt{3}$ for the sound speed in the plasma. For the reference values of eq. (2.1), we find that $\chi_{\mathrm{I}}=0.16$. This means that neutrinos are created reasonably rapidly compared to the expansion timescale. The fireball's opacity to neutrinos is described in terms of the optical depth $\tau=R / \lambda$, where $R$ is the length scale and $\lambda$ is the mfp:

$$
\tau^{(\mathrm{e})}=54 \times\left(\mathscr{E}_{52}\right)^{5 / 4}\left(R_{6.5}\right)^{-11 / 4}, \quad \tau^{(\mu, \tau)}=7.4 \times\left(\mathscr{E}_{52}\right)^{5 / 4}\left(R_{6.5}\right)^{-11 / 4} .
$$

For reference initial conditions, $\tau^{(\mathrm{e}, \mu, \tau)}>1$ so that the fireball is opaque to neutrinos of all flavors.

\section{Evolution of the neutrino fireball}

Figure 1 shows how the parameter space of the neutrino-fireball is divided in the regions I, II and III by the $\tau^{(\mu, \tau)}=1$ and $\tau^{(\mathrm{e})}=1$ contours. Also shown is the neutrino creation rate parameter $\chi$. In region I neutrinos are created rapidly compared to the expansion timescale while the fireball is neutrino-opaque, so that thermodynamic equilibrium will be established rapidly.

The evolution of the fireball is very similar to that of a neutrinoless fireball (see e.g. [6]). The plasma expands by radiation pressure, converting radiative energy to kinetic energy of the baryons. We assume that the expansion is adiabatic and reversible, so that $R T=$ const and $\mathscr{E} R=$ const during the expansion (see ref. [6]; note that $\mathscr{E}$ denotes the thermal energy). If one of the plasma components decouples, the temperature-radius relationship still holds but a fraction of the energy is lost at constant radius [5].

Consider the evolution of a fireball that starts with initial energy $\mathscr{E}_{0}$ and size $R_{0}$ in region I. The trajectory is sketched in figure 1. Starting from the point denoted as ' 0 ' in figure 1 , the plasma expands along a $\mathscr{E} R=\mathscr{E}_{0} R_{0}$ line until it reaches the $\tau^{(\mu, \tau)}=1$ contour, where the muonand tau-neutrinos decouple from the plasma. These carry away $14 / 43 \simeq 33 \%$ of the thermal energy 
that is then available (a fraction of the initial energy is already converted to kinetic energy of the nucleons). This moves the fireball from point 1 to point 2 . The electron-neutrinos remain in thermal equilibrium with the plasma, which expands along a $\mathscr{E} R=\mathscr{E}_{2} R_{2}$ curve. When the plasma becomes transparent to electron-neutrinos at $\tau^{(\mathrm{e})}=1$, these carry away $7 / 29 \simeq 24 \%$ of the energy (point 4).

\section{Neutrino emission}

Apart from the decoupling bursts, neutrinos are emitted continuously in regions where the creation rate is sufficiently high and the plasma is transparent to neutrinos. This continuous emission component is smaller than the bursts and never causes dramatic energy losses. The spectrum of the emitted neutrinos is thermal, with observed temperature roughly equal to the initial temperature of the plasma $[3,5]$. We find a total energy and mean energy of

$$
E_{(v \text { tot })}=3.1 \times 10^{52} \operatorname{erg}\left(\mathscr{E}_{52}^{(0)} R_{6.5}^{(0)}\right)^{11 / 16}, \quad\left\langle E_{v}\right\rangle=56 \mathrm{MeV}\left(\mathscr{E}_{52}^{(0)}\right)^{1 / 4}\left(R_{6.5}^{(0)}\right)^{-3 / 4} .
$$

Because the fireball has not expanded much in between the two decoupling events (see figure 1), the intrinsic time spread is determined by the size of the fireball at the second burst: $\Delta t \sim 0.4 \mathrm{~ms}$. Due to dispersion, the observed time spread is expected to be around $\Delta t \sim 1 \mathrm{~ms}$. Because of the relatively low mean energy and isotropic outflow, detection possibilities of such a neutrino source are limited to a few Mpc (see ref. [5] for a discussion and references).

\section{Conclusions}

We have described the physics of neutrinos in a hot fireball plasma. For parameters that are thought likely for the initial fireballs in gamma-ray bursts, we find that the dominant neutrino processes are leptonic and thus cooling is equally rapid in baryon-free fireballs. A fireball that starts in the neutrino-opaque region I creates neutrinos rapidly enough to establish thermodynamic equilibrium. The neutrinos follow the usual hydrodynamical evolution of the fireball until decoupling. This results in a ms burst of $\sim 60 \mathrm{MeV}$ neutrinos, which carry away $\sim 30 \%$ of the initial energy in the fireball. Although this is a sizable fraction, neutrino cooling is never dramatic and the neutrinos will be difficult to detect.

\section{References}

[1] Cavallo, G., Rees, M.J., 1978, MNRAS, 183, 359

[2] Dicus, D.A., 1972, Phys.Rev.D6, 941

[3] Goodman, J., 1986, ApJ, 308, L47

[4] Itoh, N., Adachi, T., Nakagawa, M., Kohyama, Y., Munakata, H., 1989, ApJ, 339, 354

[5] Koers, H.B.J., Wijers, R.A.M.J., 2005, MNRAS, 364, 934

[6] Shemi, A., Piran, T., 1990, ApJ, 365, L55

[7] Tubbs, D.L., Schramm, D.N., 1975, ApJ, 201, 467

[8] Van Paradijs, J., Kouveliotou, C., Wijers, R.A.M.J., 2000, ARA\&A, 38, 379 